CAHIER DE RECHERCHE \#1617E

Département de science économique

Faculté des sciences sociales

Université d'Ottawa
WORKING PAPER \#1617E

Department of Economics

Faculty of Social Sciences

University of Ottawa

\title{
Valuing Inputs Under Supply Uncertainty: The Bayesian Shapley Value*
}

\author{
Roland Pongou ${ }^{\dagger}$ and J ean-Baptiste Tondji ${ }^{\ddagger}$
}

October 2016

\footnotetext{
${ }^{*}$ We would like to thank Pierre Evariste Nguimkeu for his valuable comments.

${ }^{\dagger}$ Department of Economics, University of Ottawa, 120 University Private, Ottawa, Ontario, Canada, K1N 6N5; Email: rpongou@uottawa.ca.

₹ Department of Economics, University of Ottawa, 120 University Private, Ottawa, Ontario, Canada, K1N 6N5; Email: jtond063@uottawa.ca.
} 


\begin{abstract}
We consider the problem of valuing inputs in a production environment in which input supply is uncertain. Inputs can be workers in a firm, risk factors for a disease, securities in a financial market, or nodes in a networked economy. Each input takes its values from a finite set, and uncertainty is modeled as a probability distribution over this set. First, we provide an axiomatic solution to our valuation problem, defining three intuitive axioms which we use to uniquely characterize a valuation scheme that we call the a priori Shapley value.

Second, we solve the problem of valuing inputs a posteriori - that is, after observing output. This leads to the Bayesian Shapley value.

Third, we consider the problem of rationalizing uncertainty when the inputs are rational workers supplying labor in a non-cooperative production game in which payoffs are given by the Shapley wage function. We find that probability distributions over labor supply that can be supported as mixed strategy Nash equilibria always exist. We also provide an intuitive condition under which we prove the existence of a pure strategy Nash equilibrium. We present several applications of our theory to real-life situations.
\end{abstract}

Key words: Input valuation, uncertainty, a priori Shapley value, Bayesian Shapley value, rationalizability.

JEL Classification: C70, D20, D80, J30. 


\section{Introduction}

In this paper, we consider the problem of valuing inputs in a production environment where input supply is uncertain. Each input is a discrete variable taking values from a finite set, and uncertainty is formalized as a probability distribution over this set, viewed as a sample space. A production function maps each input profile to a measurable output. Given the uncertainty characterizing the supply of inputs, the level of output cannot be known a priori. We answer the following questions:

1. What value can we attach to each input a priori?

2. Assume an output level is realized. What value can we attach to each input a posteriori?

3. Assume that inputs are "rational" production factors (e.g., workers) choosing their effort level in a non-cooperative manner. Can certain probability distributions over effort levels be rationalized?

The production environment described above fits many real-life situations, as illustrated by the following examples.

Example 1. (Valuing workers' unobserved abilities and skills) The production of a certain good requires $n$ types of skills and $n$ workers, with each worker supplying a different skill. Output is generated by a production function $f$ that depends on the ability of each worker in the skill that she possesses. A worker's ability level belongs to the finite set $T=\{0,1,2\}$, where 0 means low ability, 1 medium ability, and 2 high ability. The ability of a worker is not known, but the distribution of ability for each skill type in the population is known. If we assume that each worker is randomly chosen from the pool of workers who possess her skill type, the answer to question (1) above will determine the a priori contribution of each skill type in the production process. Following the realization of an output level, we answer question (2) by first uncovering the posterior distributions of abilities among the selected workers, and using this information, we determine the effective contribution of each skill type to the production of the output. Finally, given that the ability distribution is exogenous in this particular example, question (3) is irrelevant.

Example 2. (Assessing the risk factors of a disease) A disease (e.g., diabetes) is caused by a set of risks factors (e.g., obesity, low fruit and vegetable intake, lack of physical activity, smoking, high blood pressure). The level (or severity) of each risk factor $i$ belongs to a finite sample space $T_{i}$, and the production function of the disease, $f$, maps each risk profile to the probability with which an individual with such a risk profile will develop the disease. Assuming that each risk factor has a probability distribution in the population, the answer to question (1) will reveal the total contribution of each risk factor to the prevalence of the disease in the population. Assume that the prevalence of the disease is observed in a subgroup of the population for which no information on the distribution of risk factors is available. Then one can determine the total contribution of each risk factor to the level of the disease in that subgroup by answering question (2). 
Example 3. (Rationalizing random and non-random labor supply in a firm) A firm employs $n$ workers. Each worker supplies labor in discrete units (zero hours, one hour, two hours, and so on) up to a maximum amount - say, nine hours per day. A production technology $f$ maps each profile of hours worked to a real number output that is shared among the workers according to the Shapley wage function (see below). Unlike in Example 1 and Example 2, we assume that there is no uncertainty a priori regarding the number of hours of work supplied by each worker. We are interested in whether there exist probability distributions over the number of worked hours that can be rationalized as Nash equilibria.

In this paper, we develop a theory to answer these questions. Our model of an uncertain production environment is a tuple $\left(N,\left(T_{i}\right)_{i \in N}, f, \pi\right)$, where $N$ is a finite set of inputs, $T_{i}$ a finite set of values that can be taken by input $i, f$ a production function that maps each input profile to a real number output, and $\pi=\left(\pi_{i}\right)_{i \in N}$ a profile of probability distributions - with $\pi_{i}$ being the probability distribution of input $i$ over the set $T_{i}$. We take an axiomatic approach to answer the question of how to value each input a priori. We define three intuitive axioms of a valuation scheme - probabilistic symmetry, probabilistic efficiency, and marginality - and show that one and only one valuation scheme satisfies all three axioms. We call this scheme the a priori Shapley value. These axioms generalize to our environment the traditional axioms of symmetry, efficiency, and marginality that characterize the classical Shapley value (Shapley (1953), Young (1985)). In the classical environment, it is assumed that for each input $i \in N, T_{i}=\{0,1\}, \pi_{i}(0)=0$, and $\pi_{i}(1)=1$. That is, each agent has only two options - "work" and "not work" - and all agents choose to work. ${ }^{1}$ Clearly, our environment is more general, as players have more choices and may not all choose the same action. Another distinctive feature of our environment is to assume uncertainty in the supply of inputs, which makes this environment more realistic.

Now, assuming that a level $Y$ of the output is realized, we answer the question of how to value each input a posteriori. We use a Bayesian updating procedure to determine the posterior probability distributions of inputs, $P(. \mid Y)$, which we use to obtain the Bayesian Shapley value. The Bayesian Shapley value therefore determines the contribution of each input to the realized output $Y$. This value is the unique valuation procedure that satisfies modified versions of the aforementioned axioms that take into account the updated profile of probability distributions $P(. \mid Y)$. This is because our characterization of the a priori Shapley value is valid for any profile of probability distributions $\pi$. Importantly, we also show that averaging the Bayesian Shapley value over the set of realizable outputs yields the a priori Shapley value.

In certain situations, such as when a worker's performance depends on weather conditions, the probability distribution of each input might be exogenous. But there also exist situations in which inputs - viewed as rational agents - may choose probability distributions that maximize their payoffs. We therefore answer the question of whether such probability distributions exist in our setting. We assume a production environment $\left(N,\left(T_{i}\right)_{i \in N}, f\right)$ free of any exogenous profile of probability distributions of inputs. We additionally assume

\footnotetext{
${ }^{1}$ See also Shapley and Shubik's (1967) application of the value to agrarian economies.
} 
that the payoff to each worker at each input profile is determined by the value of the Shapley wage function at that profile. ${ }^{2}$ These assumptions lead to a finite non-cooperative game $\left(N, \prod_{i=1}^{n} T_{i},\left(u_{i}\right)_{i \in N}\right)$ where $u_{i}$ is the payoff function of player $i$ given by the Shapley wage function under the technology $f$. A profile of probability distributions $\pi$ is rationalizable if it is a Nash equilibrium of the game $\left(N, \prod_{i=1}^{n} T_{i},\left(u_{i}\right)_{i \in N}\right)$. We prove the existence of rationalizable probability distributions. This proof simply derives from the proof of the existence of a mixed strategy equilibrium in any finite non-cooperative game (Nash (1951)). We also find a natural condition that guarantees the existence of a pure strategy Nash equilibrium. This result states that if the production function exhibits positive interaction or complementarity, there exists a rationalizable probability distribution in which each agent assigns the maximum weight to the highest effort level.

In addition to the applications mentioned above, we provide an application to contagion in a networked economy in which self-interested agents freely form and sever bilateral links. Rationality is captured by a certain equilibrium notion (e.g., pairwise stability, stochastic stability, etc.). We apply the a priori Shapley value to determine the expected contribution of each agent (or node) to the spread of a random infection that might hit the economy. This application assumes that only equilibrium networks can form. Second, given an observed contagion level, we are able to determine the contribution of each agent to this outcome using the Bayesian Shapley value. In particular, this application is implemented for fidelity economies (see Pongou (2010), Pongou and Serrano (2013)) to determine individual responsibilities in the spread of a random infection a priori and a posteriori. It turns out that these measures can also be used to assess the expected and the actual centrality of agents in an economy. While the expected centrality of an agent depends on his/her desired number of partners, his/her actual centrality depends on the realized equilibrium network, and both can be very different.

To the best of our knowledge, we are the first to propose an axiomatic solution to the problem of input valuation in an uncertain production environment. We are also the first to introduce the notions of the $a$ priori Shapley value and the Bayesian Shapley value. In addition, our environment may apply to several real-life situations as shown in the examples above. Theoretical and empirical works on the general transferableutility environment assume no uncertainty in the supply of inputs (see, e.g., Roth (1989) and Serrano (2013) for an excellent account of the different generalizations of the classical Shapley value). Our model differs in assuming that exogenous shocks may affect the supply of inputs.

Our work is related to papers that have generalized the Shapley value to games in which players have multiple options (see, e.g., Freixas (2005), Hsiao and Raghavan (1993)). We depart from these papers by incorporating uncertainty. Our axiomatic approach is also different. Also, our scope is different in that we introduce a non-cooperative production game with Shapley payoffs and prove the existence of mixed and pure strategy Nash equilibria. Another distinctive feature of our work is the application of the a priori and

\footnotetext{
${ }^{2}$ The value of the Shapley wage function at an input profile $x \in T=\prod_{i=1}^{n} T_{i}$ is given by the a priori Shapley value of the uncertain production environment $\left(N,\left(T_{i}\right)_{i \in N}, f, \pi\right)$ where each component of $x$ receives a weight of 1 (that is, each agent $i$ affects a weight of 1 to $x_{i}$, and affects a weight of 0 to any $y_{i} \neq x_{i}$ ).
} 
the Bayesian Shapley values to networked economies, with a particular emphasis on fidelity networks.

The rest of this paper is organized as follows. Section 2 introduces the uncertain production environment and other preliminary concepts. Section 3 proposes an axiomatic solution to the valuation problem a priori. Section 4 introduces the Bayesian Shapley value as a solution to the valuation problem a posteriori. Section 5 is devoted to the analysis of the existence of rationalizable probability distributions in a non-cooperative production game under the Shapley wage function. In Section 6, we provide a specific application of our analysis to networked economies. Section 7 concludes. All proofs are collected in the appendix.

\section{Uncertain Production Environment}

An uncertain production environment is modeled as a tuple $\mathcal{P}=\left(N,\left(T_{i}\right)_{i \in N}, f, \pi\right)$ where $N=\{1,2, \ldots, n\}$ is a finite set of inputs of cardinality $n, T_{i}=\left\{0,1,2, \ldots, t_{i}\right\}$ is a finite set of values an input $i$ can take, $f$ is a production function which maps an input profile $x=\left(x_{1}, \ldots, x_{n}\right) \in T=\prod_{i=1}^{n} T_{i}$ to a real number output $f(x)$, and $\pi=\left(\pi_{i}\right)_{i \in N}$ is a profile of probability distributions of inputs. More formally, for each input $i$, we have:

$$
\begin{aligned}
\pi_{i}: T_{i} & \longrightarrow[0,1] \\
x_{i} & \longmapsto \pi_{i}\left(x_{i}\right) \text { with } \sum_{x_{i} \in T_{i}} \pi_{i}\left(x_{i}\right)=1 .
\end{aligned}
$$

Throughout the paper, we assume $f(0,0, \ldots, 0)=0$, which means that if all inputs are inactive, there will be no output. The interpretation of inputs depends on the production environment being analyzed. For example, an input level may be the number of hours supplied by a worker in a firm, the unobserved ability of a worker, the level of effort that a student puts into a class project, or the severity of a risk factor for a disease. Note that the elements of each input set $T_{i}$ need not be interpreted as numbers - they can simply be viewed as labels. For instance, $T_{i}$ might represent the different types of jobs to which worker $i$ can be assigned in a firm; in this case, $T_{i}$ is not necessarily an ordered set. We also note that, for each input $i, T_{i}$ might represent a different set of objects (that is, action 1 in set $T_{1}$ might not represent the same object as action 1 in set $T_{2}$ ).

We denote by $\pi(x)$ the probability of the realization of an input profile $x \in T$. Interestingly, if we assume that the input variables are independently distributed, the probability of the realization of an input profile $x \in T$ is:

$$
\pi(x)=\prod_{i \in N} \pi_{i}\left(x_{i}\right)
$$

In the next section, we answer the question of how to value each input factor a priori in an uncertain production environment. 


\section{A Priori Shapley Value}

Given a production environment $\mathcal{P}=\left(N,\left(T_{i}\right)_{i \in N}, f, \pi\right)$, what is the contribution a priori of each input factor to output production? Answering this question is important in several contexts. It may reveal the pay a worker employed by a firm can expect to receive from the firm's revenue, or the fraction of the prevalence of a disease that can be attributed to a given risk factor. We propose an axiomatic solution to this problem. We introduce three intuitive axioms inspired by the works of Shapley (1953) and Young (1985), and use them to uniquely characterize a valuation procedure that we call the a priori Shapley value. The following definition of the notion of probabilistic symmetry will be needed in the statement of one of these axioms.

Definition 1. Let $\mathcal{P}=\left(N,\left(T_{i}\right)_{i \in N}, f, \pi\right)$ be an uncertain production environment. Two input factors $i$ and $j$ are said to be probabilistically symmetric if: (1) $\pi_{i}=\pi_{j}$; and (2) for any input profile $x$ such that $x_{i}=0$ or $x_{j}=0, f(x)=f\left(\tau_{i j}(x)\right)$, where $\tau_{i j}(x)=x-x_{i} e_{i}+x_{j} e_{i}-x_{j} e_{j}+x_{i} e_{j}$, with $e_{k}=(0, \ldots, 0,1,0, \ldots 0), k \in\{i, j\}$, being the $n$-component unit vector whose $k^{\text {th }}$ component is one and all of the other components are zero.

Two inputs are probabilistically symmetric if they have the same probability distribution and if permuting their levels in the production process does not change the output.

The following definition of a valuation procedure is also needed.

Definition 2. A valuation procedure is a function $\phi$ which maps any production environment $\mathcal{P}=\left(N,\left(T_{i}\right)_{i \in N}, f, \pi\right)$ to a vector $\left(\phi_{i}(\mathcal{P})\right)_{i \in N}$, where $\phi_{i}(\mathcal{P})$ is a real number representing the value of input $i$. If no ambiguity is possible, we will denote $\phi_{i}(\mathcal{P})$ by $\phi_{i}(f)$.

We also need the following notation.

Notation 1. Let $x$ be an input profile. We denote by $|x|=\left|\left\{i \in N: x_{i}>0\right\}\right|$ the number of active inputs at $x$. We also define the following binary relations $\unlhd$ and $\triangleleft$ on the set of all the possible input profiles of an uncertain production environment $\mathcal{P}=\left(N,\left(T_{i}\right)_{i \in N}, f, \pi\right)$ as follows: for any input profiles $a, x \in T$, $[a \unlhd x]$ if and only if $\left[a_{i} \neq x_{i} \Rightarrow a_{i}=0\right]$; and $[a \triangleleft x]$ if and only if $[a \unlhd x$ and $a \neq x]$. For example, $(0,1,4,0, \ldots .9) \triangleleft(1,1,4,2, \ldots, 9)$. Moreover, for $i \in N$ and $a, x \in T$, the notation $a \triangleleft_{0}^{i} x$ means that $a \triangleleft x$ and $a_{i}=0$.

\subsection{Axioms and Characterization}

We now present the axioms.

Axiom 1. (Probabilistic symmetry)

A valuation procedure $\phi$ is probabilistically symmetric if for any production environment $\mathcal{P}=\left(N,\left(T_{i}\right)_{i \in N}, f, \pi\right)$ and any probabilistically symmetric input factors $i$ and $j, \phi_{i}(f)=\phi_{j}(f)$. 


\section{Axiom 2. (Probabilistic efficiency)}

$A$ valuation procedure $\phi$ is probabilistically efficient if for any production environment $\mathcal{P}=\left(N,\left(T_{i}\right)_{i \in N}, f, \pi\right)$, $\sum_{i \in N} \phi_{i}(f)=\sum_{x \in T} \pi(x) f(x)$.

Axiom 3. (Marginality)

A valuation procedure $\phi$ satisfies the marginality axiom if for any production environments $\mathcal{P}=\left(N,\left(T_{i}\right)_{i \in N}, f, \pi\right)$ and $\mathcal{P}^{\prime}=\left(N,\left(T_{i}\right)_{i \in N}, g, \pi\right)$, all $i \in N$, and all $x, a \in T$ such that $a \triangleleft_{0}^{i} x,\left[f\left(a+x_{i} e_{i}\right)-f(a) \geq g\left(a+x_{i} e_{i}\right)-\right.$ $g(a)] \Rightarrow\left[\phi_{i}(f) \geq \phi_{i}(g)\right]$.

The probabilistic symmetry axiom states that probabilistically interchangeable inputs should be identically valued a priori. The probabilistic efficiency axiom says that the expected output of the production environment should be entirely shared among the different inputs. The marginality axiom states that, if there is a change in the technology that increases the marginal productivity of an input, then the valuation procedure should value that input more under the new technology.

We now state the main result of this section.

Theorem 1. One and only one valuation procedure satisfies simultaneously the axioms of probabilistic efficiency, probabilistic symmetry, and marginality. This valuation procedure is defined, for every uncertain production environment $\mathcal{P}=\left(N,\left(T_{i}\right)_{i \in N}, f, \pi\right)$ and every input $i \in N$, by:

$$
A S V_{i}(\mathcal{P})=\sum_{x \in T} \pi(x)\left\{\sum_{a \triangleleft_{0}^{i} x} \frac{(|a|) !(|x|-|a|-1) !}{(|x|) !}\left[f\left(a+x_{i} e_{i}\right)-f(a)\right]\right\} .
$$

We call this valuation procedure the a priori Shapley value.

For clarity, the proofs of this and all the subsequent results are provided in the appendix. Before we proceed further, we would like to explain the function $A S V_{i}(\mathcal{P})$.

Interpretation 1. Without loss of generality, assume that $\mathcal{P}$ is a firm in which $n$ workers supply labor with uncertainty. The set of all the feasible input profiles is $T$. A profile of hours worked $x$ is supplied with probability $\pi(x)$, yielding output $f(x)$. To understand the function $A S V_{i}(\mathcal{P})$, assume that, at each profile $x$, workers enter the production process in some random order, and that all the $(|x|)$ ! orderings of those who supply a positive amount of work are equally likely. In fact, we can suppose there are $|x|$ active positions in the firm at $x$, and that each worker occupies his position in $x$ once he enters the firm. Starting at a profile $a$ such that $a \unlhd x$, when a worker $i$ who initially satisfies $a_{i}=0$ enters the firm to find the other active workers $S=\left\{k \in N: a_{k}=x_{k}>0\right\}$, he is assigned the position $x_{i}$. It follows that the fraction $\frac{(|a|) !(|x|-|a|-1) !}{(|x|) !}$ represents the probability that a given worker $i$, with $a_{i}=0$, joins a subgroup of active workers $S$. When worker $i$ joins $S$, the new input profile is $a+x_{i} e_{i}$ and the output is $f\left(a+x_{i} e_{i}\right)$; so his marginal contribution is $f\left(a+x_{i} e_{i}\right)-f(a)$. The number $\sum_{a \triangleleft_{0}^{i} x} \frac{(|a|) !(|x|-|a|-1) !}{(|x|) !}\left[f\left(a+x_{i} e_{i}\right)-f(a)\right]$ is therefore the expected marginal contribution of $i$ when the profile $x$ is realized. Since $x$ occurs with probability $\pi(x)$, the expected payoff of $i$ 
is the average of his expected marginal contribution over the set of profiles $T$, which is his a priori Shapley value $A S V_{i}$ in $\mathcal{P}$.

Throughout the paper, to simplify notation in some sections and in the proofs, we will denote $\frac{(|a|) !(|x|-|a|-1) !}{(|x|) !}$ by $\varphi(a, x)$ and the marginal contribution $f\left(a+x_{i} e_{i}\right)-f(a)$ of input $i$ in profile $a+x_{i} e_{i}$ such that $a_{i}=0$ by $m c(i, f, a, x)$.

We note two important remarks:

1. If a production environment $\mathcal{P}$ is such that an input profile $x$ is realized with certainty $($ i.e., $\pi(x)=1)$, then for each input $i$, the a priori Shapley value becomes:

$$
A S V_{i}(\mathcal{P})=A S V_{i}(x)=\sum_{a \triangleleft_{0}^{i} x} \frac{(|a|) !(|x|-|a|-1) !}{(|x|) !}\left[f\left(a+x_{i} e_{i}\right)-f(a)\right]
$$

We call $A S V_{i}(x)$ the Shapley wage function. In fact, in an environment $\mathcal{F}=\left(N,\left(T_{i}\right)_{i \in N}, f\right)$ in which uncertainty is removed, $A S V_{i}(x)$ defines a real-valued function on the set of input profiles $T$. This function was first defined in Aguiar et al. (2016), who study a different problem than the one addressed in this paper. It follows that, in an uncertain production environment $\mathcal{P}=\left(N,\left(T_{i}\right)_{i \in N}, f, \pi\right)$, the $a$ priori Shapley value of an input $i$ is simply the weighted average of its Shapley wage over the set of input profiles:

$$
A S V_{i}(\mathcal{P})=\sum_{x \in T} \pi(x)\left\{A S V_{i}(x)\right\}
$$

2. As a second remark, we note that the a priori Shapley value generalizes the classical Shapley value. In fact, the transferable-utility environment in which the classical Shapley value was first defined can be modeled as a tuple $\mathcal{P}=\left(N,\left(T_{i}\right)_{i \in N}, f, \pi\right)$, where $T_{i}=\{0,1\}$ for each $i, f$ is a characteristic function defined on $T$, and $\pi=\left(\pi_{i}\right)_{i \in N}$ with $\pi_{i}(0)=0$ and $\pi_{i}(1)=1$ for each player $i$ (meaning that the grand coalition forms). It follows that $\pi((1, \ldots, 1))=1$. In fact, notice that an input profile $x$ is equivalent to a coalition $S(x)=\left\{i \in N: x_{i}=1\right\}$ of inputs whose level is $1 \mathrm{in} x$. In addition, noticing that in the formula of the a priori Shapley wage function, $a \triangleleft_{0}^{i} x$ for $a, x \in T$ means that $S(a) \subset S(x)=N$ and $i \notin S(a)$, and $a+x_{i} e_{i}$ is equivalent to $S(a) \cup\{i\}$, we immediately find that:

$$
A S V_{i}((1, \ldots, 1))=\sum_{S \subset N, i \notin S} \frac{(|S|) !(n-|S|-1) !}{(n) !}[f(S \cup\{i\})-f(S)]
$$

which is the classical Shapley value. Our value is more general not only because we incorporate uncertainty, but also because even when players choose with certainty a level of input, each player may make a different choice. In this sense, even if we restrict ourselves to the classical environment where each player has a binary choice, our value is still more general in that we do not impose that all players choose the input level 1 as in Shapley (1953). 


\subsection{Some Illustrative Examples}

In this section, we present some examples to illustrate the a priori Shapley value.

Example 4. (Firm and suppliers) An economy consists of a firm and two suppliers $A$ and $B$ who respectively supply inputs $x_{A}$ and $x_{B}$ to the firm, with $x_{A} \in\{0,4,9\}$ and $x_{B} \in\{0,9\}$. The firm's technology is represented by the constant elasticity of substitution production function $g\left(x_{A}, x_{B}\right)=100\left(\frac{1}{3} x_{A}^{1 / 2}+\frac{2}{3} x_{B}^{1 / 2}\right)^{1 / 2}$. Supplier A provides 0 units of commodity $x_{A}$ with probability 0.25, 4 units of this commodity with probability 0.5, and 9 units with probability 0.25. Supplier B provides 0 units of commodity $x_{B}$ with probability 0.25, and 9 units with probability 0.75. We also assume that the price of each output produced is equal to 1 , so that for an input profile $x=\left(x_{A}, x_{B}\right), g(x)$ also represents the revenue of the output generated by $x$. Table 1 shows the probability of realizing each profile of inputs. Table 2 presents the production function of the firm, and Table 3 presents the a priori Shapley value of the two suppliers for each input profile.

Table 1: Joint probability of input profiles

\begin{tabular}{|c|c|c|c|c|c|}
\hline$x=\left(x_{A}, x_{B}\right)$ & $\pi(x)$ & $x=\left(x_{A}, x_{B}\right)$ & $\pi(x)$ & $x=\left(x_{A}, x_{B}\right)$ & $\pi(x)$ \\
\hline$(0,0)$ & 0.0625 & $(4,0)$ & 0.125 & $(9,0)$ & 0.0625 \\
\hline$(0,9)$ & 0.1875 & $(4,9)$ & 0.375 & $(9,9)$ & 0.1875 \\
\hline
\end{tabular}

Table 2 : Production function $g$

\begin{tabular}{|c|c|c|c|c|c|}
\hline$x=\left(x_{A}, x_{B}\right)$ & $g(x)$ & $x=\left(x_{A}, x_{B}\right)$ & $g(x)$ & $x=\left(x_{A}, x_{B}\right)$ & $g(x)$ \\
\hline$(0,0)$ & 0 & $(4,0)$ & 81.65 & $(9,0)$ & 100 \\
\hline$(0,9)$ & 141.42 & $(4,9)$ & 163.3 & $(9,9)$ & 173.20 \\
\hline
\end{tabular}

Table 3: The a priori Shapley value $A S V(x)=\left(A S V_{A}(x), A S V_{B}(x)\right)$

\begin{tabular}{|c|c|c|c|}
\hline$x_{B} \downarrow x_{A} \rightarrow$ & 0 & 4 & 9 \\
\hline 0 & $(0,0)$ & $(81.65,0)$ & $(100,0)$ \\
\hline 9 & $(0,141.42)$ & $(51.765,111.535)$ & $(65.89,107.31)$ \\
\hline
\end{tabular}

Using the information in Tables 1-3, we derive the a priori Shapley value for the production environment $\mathcal{P}=\left(N,\left(T_{A}, T_{B}\right), g, \pi\right)\left(N=\{A, B\}, T_{A}=\{0,4,9\}, T_{B}=\{0,9\}\right.$, and $\left.T=T_{A} \times T_{B}\right)$ as follows:

$$
A S V_{A}(\mathcal{P})=\sum_{x \in T} \pi(x) A S V_{A}(x)=48.2225 \text { and } A S V_{B}(\mathcal{P})=\sum_{x \in T} \pi(x) A S V_{B}(x)=88.4625
$$

Each supplier is therefore expected to have a different effect on the output of the firm. This is for three reasons. The first is that the suppliers are not interchangeable in the production process, as illustrated by Table 2. The second reason is that they do not supply inputs with the same probability, and the third is that input $x_{A}$ has a smaller effect on the production of the output, as illustrated by the production function. 
The next example shows how the a priori Shapley value can be applied to a financial system of banks (see, e.g., Allen and Gale (2000), Battiston et al.(2012), Lin (2016)) in order to measure the contribution of each bank to systemic risk. ${ }^{3}$

Example 5. (Measuring financial risk) Assume a financial market in which banks are connected and investments are highly correlated. For simplicity, assume that there are only two banks $b_{1}$ and $b_{2}$ which have up to two choices of risk investments. Each bank may decide to avoid any risk investment - which corresponds to choice 0 - or to invest some part of its assets in the risky activities, corresponding to choice 1. A global risk function $h$ maps a vector of investment choices to a risk measure. The probability distribution $\pi$ over the set of choices $I_{A}=\{0,1\}=I_{B}$ is given as follows: $\pi_{b_{1}}(0)=0.6, \pi_{b_{1}}(1)=0.4, \pi_{b_{2}}(0)=0.25$, and $\pi_{b_{2}}(1)=0.75$. The risk measure function $h$ is given by:

$$
h(i)=\left\{\begin{array}{lll}
1 & \text { if } & i \neq(0,0) \\
0 & \text { if } & i=(0,0)
\end{array}\right.
$$

The a priori Shapley value of each bank is given in Table 4 below:

Table 4: The a priori Shapley value of banks $\left(A S V(i)=\left(A S V_{b_{1}}(i), A S V_{b_{2}}(i)\right)\right)$

\begin{tabular}{|c|c|c|}
\hline Level of risk investment & 0 & 1 \\
\hline 0 & $(0,0)$ & $(0,1)$ \\
\hline 1 & $(1,0)$ & $\left(\frac{1}{2}, \frac{1}{2}\right)$ \\
\hline
\end{tabular}

The a priori Shapley value $A S V(x)$ measures the contribution of each bank to systemic risk at investment profile $x$. The expected contribution of each bank to systemic risk in the uncertain environment defined by $\mathcal{P}=\left(N=\left\{b_{1}, b_{2}\right\},\left(I_{A}, I_{B}\right), h, \pi\right)$ is given hereunder:

$$
A S V_{b_{1}}(\mathcal{P})=0.25 \text { and } A S V_{b_{2}}(\mathcal{P})=0.6
$$

These values illustrate the fact that banks that are more likely to invest in risky activities contribute more to the failure of the overall system.

\section{The Bayesian Shapley Value}

This section is devoted to the problem of valuing inputs a posteriori; that is, after the realization of the output. Let $\mathcal{P}=\left(N,\left(T_{i}\right)_{i \in N}, f, \pi\right)$ be an uncertain production environment. Assume that an output level $Y$ is realized. Given the prior probability distribution $\pi_{i}$ of each input variable $i$, we can derive the posterior probability distributions using Bayesian updating. We then apply these posterior probability distributions to obtain what we call the Bayesian Shapley value, and we provide an axiomatic characterization of this value.

\footnotetext{
${ }^{3}$ See Section 5 too for an application to networked economies.
} 
To illustrate, imagine a firm in which the manager observes the level of output $Y$, but cannot observe the inputs (or ability) of workers. The realized output has to be shared among the workers. What share of this output should the manager give each worker? In answering this question, we assume that the manager is Bayesian and that she has prior beliefs about the distribution of inputs (that is, she knows $\pi$ ).

Consider a simple case where there are exactly three input profiles $x, y$ and $z$ such that $f(x)=f(y)=$ $f(z)=Y$. The prior probabilities of these profiles are $\pi(x), \pi(y)$ and $\pi(z)$, respectively. The prior probability of the realization of $Y$ is therefore:

$$
P(Y)=\pi(x) P(Y \mid x)+\pi(y) P(Y \mid y)+\pi(z) P(Y \mid z) .
$$

Since $P(Y \mid x)=P(Y \mid y)=P(Y \mid z)=1$, it follows that $P(Y)=\pi(x)+\pi(y)+\pi(z)$. We note that this sum is not necessarily equal to 1 . Using Bayes' theorem, the posterior probability that we are looking for is obtained by dividing the prior probability by $P(Y)$. For each $h \in\{x, y, z\}$, we then have:

$$
\begin{aligned}
P(h \mid Y) & =\frac{P(Y \cap h)}{P(Y)} \\
& =\frac{P(h) \times P(Y \mid h)}{P(Y)} \\
& =\frac{\pi(h)}{\pi(x)+\pi(y)+\pi(z)}, \text { since } P(h)=\pi(h) \text { and } P(Y \mid h)=1 .
\end{aligned}
$$

Knowing the posterior probability distribution $\{P(h \mid Y)\}$ over the set $\{x, y, z\}$, the average value $(A V)$ of input $i$ is:

$$
\begin{aligned}
A V_{i}(Y) & =A S V_{i}(x) P(x \mid Y)+A S V_{i}(y) P(y \mid Y)+A S V_{i}(x) P(z \mid Y) \\
& =\frac{\pi(x) A S V_{i}(x)+\pi(y) A S V_{i}(y)+\pi(z) A S V_{i}(z)}{\pi(x)+\pi(y)+\pi(z)} .
\end{aligned}
$$

We will call this valuation scheme the Bayesian Shapley value. Below, we generalize this scheme to any uncertain production environment. Let $\mathcal{P}=\left(N,\left(T_{i}\right)_{i \in N}, f, \pi\right)$ be an uncertain production environment. Denote by $\mathcal{R}_{f}=\{Y \in \mathbb{R}: \exists x \in T, f(x)=Y\}$ the set of realizable outputs. For any $Y \in \mathcal{R}_{f}$, define by $Y_{f}=\{x \in T: f(x)=Y\}$ the set of input profiles that produce $Y$. We have the following definition.

Definition 3. The Bayesian Shapley value is the function $B S A$ defined, for any uncertain production environment $\mathcal{P}=\left(N,\left(T_{i}\right)_{i \in N}, f, \pi\right)$ and any realizable output $Y \in \mathcal{R}_{f}$, by:

$$
B S A_{i}(Y)=\frac{\sum_{x \in Y_{f}} \pi(x) A S V_{i}(x)}{\sum_{x \in Y_{f}} \pi(x)}, i \in N .
$$

We now want to present an axiomatic characterization of this value. A few preliminary notions will be needed. For any uncertain production environment $\mathcal{P}=\left(N,\left(T_{i}\right)_{i \in N}, f, \pi\right)$ and any realizable output $Y \in \mathcal{R}_{f}$, define by $\mathcal{P}_{Y}=\left(N,\left(T_{i}\right)_{i \in N}, f, P(. \mid Y)\right)$ the posterior production environment associated to $Y$, where $P(. \mid Y)$ 
is the posterior probability distribution over input profiles and is defined as follows:

$$
P(h \mid Y)=\left\{\begin{array}{clll}
\frac{\pi(h)}{\sum_{x \in Y_{f}} \pi(x)} & \text { if } & h \in Y_{f} \\
0 & \text { if } & h \notin Y_{f}
\end{array}\right.
$$

We also define the notion of symmetry a posteriori.

Definition 4. Let $\mathcal{P}=\left(N,\left(T_{i}\right)_{i \in N}, f, \pi\right)$ be an uncertain production environment and $Y \in \mathcal{R}_{f}$ a realizable output. Two input factors $i$ and $j$ are said to be symmetric a posteriori if for any input profile $x \in Y_{f}$ such that $x_{i}=0$ or $x_{j}=0, f(x)=f\left(\tau_{i j}(x)\right)$.

We now present the axioms.

\section{Axiom 4. (Symmetry a posteriori)}

$A$ valuation procedure $\phi$ is symmetric a posteriori if for any production environment $\mathcal{P}=\left(N,\left(T_{i}\right)_{i \in N}, f, \pi\right)$, any realizable output $Y \in \mathcal{R}_{f}$, and any input factors $i$ and $j$ that are symmetric a posteriori, $\phi_{i}\left(\mathcal{P}_{Y}\right)=$ $\phi_{j}\left(\mathcal{P}_{Y}\right)$.

\section{Axiom 5. (Efficiency a posteriori)}

$A$ valuation procedure $\phi$ is efficient a posteriori if for any production environment $\mathcal{P}=\left(N,\left(T_{i}\right)_{i \in N}, f, \pi\right)$ and any realizable output $Y \in \mathcal{R}_{f}, \sum_{i \in N} \phi_{i}\left(\mathcal{P}_{Y}\right)=Y$.

\section{Axiom 6. (Marginality a posteriori)}

$A$ valuation procedure $\phi$ satisfies the axiom of marginality a posteriori if for any production environment $\mathcal{P}=$ $\left(N,\left(T_{i}\right)_{i \in N}, f, \pi\right)$, any realizable output $Y \in \mathcal{R}_{f}$, any production environment $\mathcal{P}^{\prime}=\left(N,\left(T_{i}\right)_{i \in N}, g, P(. \mid Y)\right)$, any input $i \in N$, and any input profiles $x \in Y_{f}$ and $a \in T$ such that $a \triangleleft_{0}^{i} x$ :

$\left[g\left(a+x_{i} e_{i}\right)-g(a) \geq f\left(a+x_{i} e_{i}\right)-f(a)\right] \Rightarrow\left[\phi_{i}\left(\mathcal{P}^{\prime}\right) \geq \phi_{i}\left(\mathcal{P}_{Y}\right)\right]$.

The axiom of symmetry a posteriori states that inputs that are interchangeable a posteriori should be identically valued. The axiom of efficiency a posteriori states that any realized output $Y$ of an uncertain production environment should be entirely shared among the different inputs. The axiom of marginality $a$ posteriori states that, if there is a change in the technology that increases the marginal productivity of an input in the posterior production environment of an uncertain production environment, then the valuation procedure should give a greater value to that input under the new technology.

The theorem below states that the Bayesian Shapley value is the only valuation scheme that satisfies all three axioms above.

Theorem 2. The Bayesian Shapley value is the only valuation procedure that satisfies simultaneously the axioms of symmetry a posteriori, efficiency a posteriori, and marginality a posteriori.

The proof of this result follows the same reasoning as the proof of Theorem 1 and is consequently omitted.

The next result shows that the average Bayesian Shapley value, which we denote by $U$, is simply equal to the a priori Shapley value. 
Theorem 3. For any uncertain production environment $\mathcal{P}=\left(N,\left(T_{i}\right)_{i \in N}, f, \pi\right)$ and any input $i \in N$, we have:

$$
U_{i}(f)=\sum_{Y \in \mathcal{R}_{f}} P(Y) B S A_{i}(Y)=\sum_{Y \in \mathcal{R}_{f}}\left\{\sum_{x \in Y_{f}} \pi(x) A S V_{i}(x)\right\}=A S V_{i}(f) .
$$

The following example applies the notion of the Bayesian Shapley value to an epidemiological context.

Example 6. (Assessing the risk factors of a disease) A disease (e.g., type 2 diabetes) is caused by a set of four leading behavioral and dietary risk factors which are: low fruit and vegetable intake (risk factor 1), smoking (risk factor 2), high body mass index (risk factor 3), and high blood pressure (risk factor 4). Let $T_{i}=\{0,1\}$ be the set of values that each risk factor $i$ can take, and $f$ the production function of the disease. The production function, given in Table 5 below, determines the probability (in percentage terms) that an individual with a given profile will have the disease. For example, if an individual has the risk profile $(1,0,0,0)$ - that is, the individual has low fruit and vegetable intake and no other risks - his probability of getting the disease is 5\%. Also, an individual who has the risk profile $(1,1,0,1)$ - that is, the individual who has low fruit and vegetable intake, smokes, and has high blood pressure - has a $30 \%$ chance of getting the disease.

For each risk profile $r=\left(r_{1}, r_{2}, r_{3}, r_{4}\right) \in T=\prod_{i=1}^{4} T_{i}$, we compute the contribution of each risk factor $i$ to the probability of getting the disease. The results are presented in Table 5. Remark that these contributions sum to $f(r)$ for each risk profile $r$.

Table 5: Values of $f(r)$ and $A S V(r)$

\begin{tabular}{|c|c|c|c|c|c|}
\hline$r$ & $f(r)$ & $A S V(r)$ & $r$ & $f(r)$ & $A S V(r)$ \\
\hline$(0,0,0,0)$ & 0 & $(0,0,0,0)$ & $(1,0,0,0)$ & 5 & $(5,0,0,0)$ \\
\hline$(0,0,0,1)$ & 20 & $(0,0,0,20)$ & $(0,1,0,1)$ & 25 & $(0,5,0,20)$ \\
\hline$(0,0,1,0)$ & 10 & $(0,0,10,0)$ & $(0,0,1,1)$ & 30 & $(0,0,10,20)$ \\
\hline$(0,1,0,0)$ & 5 & $(0,5,0,0)$ & $(0,1,1,0)$ & 13 & $(0,4,9,0)$ \\
\hline$(1,0,0,1)$ & 25 & $(5,0,0,20)$ & $(1,0,1,1)$ & 50 & $\left(\frac{60}{6}, 0, \frac{90}{6}, \frac{150}{6}\right)$ \\
\hline$(1,1,0,0)$ & 7 & $(3.5,3.5,0,0)$ & $(1,1,0,1)$ & 30 & $\left(\frac{27}{6}, \frac{27}{6}, 0, \frac{126}{6}\right)$ \\
\hline$(1,0,1,0)$ & 15 & $(5,0,10,0)$ & $(1,1,1,0)$ & 20 & $\left(\frac{31}{6}, \frac{25}{6}, \frac{64}{6}, 0\right)$ \\
\hline$(0,1,1,1)$ & 50 & $\left(0, \frac{58}{6}, \frac{88}{6}, \frac{154}{6}\right)$ & $(1,1,1,1)$ & 70 & $\left(\frac{238}{24}, \frac{230}{24}, \frac{482}{24}, \frac{730}{24}\right)$ \\
\hline
\end{tabular}

We now assume that each risk factor is uniformly distributed over $T_{i}$. That is, $\pi_{i}(0)=\pi_{i}(1)=0.5$ for each risk factor $i \in N=\{1,2,3,4\}$. The prior Shapley value of each risk factor $i$ is $A S V_{i}(\mathcal{P})=\frac{1}{16} \sum_{r \in R} A S V_{i}(r)$. We therefore have:

$$
A S V_{1}(\mathcal{P})=3.005, A S V_{2}(\mathcal{P})=2.838, A S V_{3}(\mathcal{P})=6.213, \text { and } A S V_{4}(\mathcal{P})=11.38
$$

Assume that the social planner observes that the prevalence of the disease is 50\% in a subgroup of the population and asks a Bayesian epidemiologist to compute the contribution of each risk factor to the level of 
the disease in that subgroup. The goal is to identify those risk factors in which society can invest in order to reduce the prevalence of the disease.

Based on the prior information concerning the outcome of each risk profile, there exist only two risk profiles that lead to a prevalence of $50 \%: r=(0,1,1,1)$ and $r^{\prime}=(1,0,1,1)$. The Bayesian Shapley value for each risk factor $i$ is therefore given by:

$$
B S A_{i}=\frac{\pi(r) A S V_{i}(r)+\pi\left(r^{\prime}\right) A S V_{i}\left(r^{\prime}\right)}{\pi(r)+\pi\left(r^{\prime}\right)}=\frac{1}{2}\left(A S V_{i}(r)+A S V_{i}\left(r^{\prime}\right)\right), \text { for } i \in\{1,2,3,4\} .
$$

Using the information above, we have : $B S A_{1}=5, B S A_{2}=4.83, B S A_{3}=14.83$, and $B S A_{4}=25.34$. High blood pressure is therefore the most significant contributor to the disease, as it accounts for more than half of its prevalence in that population subgroup. It is followed by high BMI, low fruit and vegetable intake, and smoking.

\section{Rationalizable Probability Distributions}

So far, we have considered a production environment $\mathcal{P}=\left(N,\left(T_{i}\right)_{i \in N}, f, \pi\right)$ where the supply of input factors is uncertain, with the uncertainty captured by an exogenous profile of the probability distributions $\pi$. This model can be justified in real-life situations where exogenous shocks affect the supply of inputs, such as when the performance of a worker depends on weather conditions. But there also exist situations in which inputs, viewed as rational players, may choose probability distributions that maximize their payoffs. In this section we ask whether certain probability distributions can be rationalized.

In order to answer this question, we consider a modified production environment $\left(N,\left(T_{i}\right)_{i \in N}, f\right)$ without uncertainty, and we associate to this environment a finite strategic form game $\mathcal{G}=\left(N, T,\left(u_{i}\right)_{i \in N}\right)$ where the payoff functions $u_{i}(x)$ are given by the Shapley wage functions $A S V_{i}(x)$ under the technology $f .{ }^{4}$

Here, each player $i$ chooses from his strategy set $T_{i}$ the amount of labor he wishes to supply to the firm. A profile $x=\left(x_{1}, x_{2}, \ldots, x_{n}\right) \in T$ (recall that $\left.T=\prod_{i=1}^{n} T_{i}\right)$ of effort levels is a profile of pure strategies. Instead of choosing a single action, players can also randomize over the set of available actions according to some probability distribution similar to $\pi$ under the uncertain production environment $\mathcal{P}$. Such a behavior would define a mixed strategy profile.

For each player $i$, we denote by $S_{i}=S_{i}\left(T_{i}\right)$ the set of all probability distributions over $T_{i}$. The set $S=S_{1} \times S_{2} \times \ldots \times S_{n}$ is therefore the set of mixed strategy profiles. Let $s_{i} \in S_{i}$ be a mixed strategy of a player $i$. By $s_{i}\left(x_{i}\right)$, we denote the probability with which action $x_{i}$ is played under the mixed strategy $s_{i} \in S_{i}$. The subset of pure strategies that are assigned positive probability by the mixed strategy $s_{i}$ is called the support of $s_{i}$. A pure strategy is a special case of a mixed strategy in which the support is a singleton.

Definition 5. A profile of probability distributions $s$ is said to be rationalizable in an environment $\left(N,\left(T_{i}\right)_{i \in N}, f\right)$ if $s$ is a Nash equilibrium of the associated strategic form game $\left(N, T,\left(u_{i}\right)_{i \in N}\right)$, where

\footnotetext{
${ }^{4}$ see Pongou and Tondji (2016) for a general definition of non-cooperative games with Shapley payoffs.
} 
$u_{i}(x)=A S V_{i}(x)$ for every $x \in T$.

Let $\left(N,\left(T_{i}\right)_{i \in N}, f\right)$ be an environment and $s=\left(s_{1}, s_{2}, \ldots, s_{n}\right)$ a mixed strategy profile of the associated game $\mathcal{G}$. Then the tuple $\mathcal{P}^{s}=\left(N,\left(T_{i}\right)_{i \in N}, f, s\right)$ is an uncertain production environment. The expected payoff of each player in $\mathcal{P}^{s}$ is also his expected payoff in the game $\mathcal{G}$ when $s$ is played. This expected payoff is:

$$
u_{i}(s)=A S V_{i}\left(\mathcal{P}^{s}\right)=\sum_{x \in T} s(x)\left\{\sum_{a \triangleleft_{0}^{i} x} \frac{(|a|) !(|x|-|a|-1) !}{(|x|) !}\left[f\left(a+x_{i} e_{i}\right)-f(a)\right]\right\} ; \text { with } s(x)=\prod_{j \in N} s_{j}\left(x_{j}\right) .
$$

In the case of pure strategies, meaning that a profile $x$ occurs with certainty, the utility of player $i$ at $x$ is:

$$
u_{i}(x)=A S V_{i}(x)=\sum_{a \triangleleft_{0}^{i} x} \frac{(|a|) !(|x|-|a|-1) !}{(|x|) !}\left[f\left(a+x_{i} e_{i}\right)-f(a)\right] .
$$

We recall the following definitions:

Definition 6. Let $\mathcal{G}$ be the game associated to a production environment $\left(N,\left(T_{i}\right)_{i \in N}, f\right)$. A player $i$ 's best response to a strategy profile $s_{-i}$ is a mixed strategy $s_{i}^{*} \in S_{i}$ such that $u_{i}\left(s_{-i}, s_{i}^{*}\right) \geq u_{i}\left(s_{-i}, s_{i}\right)$ for all strategies $s_{i} \in S_{i}$.

Definition 7. A strategy profile $s^{*}=\left(s_{1}^{*}, s_{2}^{*}, \ldots, s_{n}^{*}\right)$ is a Nash Equilibrium of a game $\left(N, T,\left(u_{i}\right)_{i \in N}\right)$ if, for all player $i, s_{i}^{*}$ is a best response to $s_{-i}^{*}$.

Equivalently, $s^{*}=\left(s_{1}^{*}, s_{2}^{*}, \ldots, s_{n}^{*}\right)$ is a Nash Equilibrium of a game $\left(N, T,\left(u_{i}\right)_{i \in N}\right)$ if and only if for every player $i$ :

$$
u_{i}\left(s^{*}\right)=\max _{\text {all } s_{i}^{\prime} s}\left\{u_{i}\left(s_{-i}^{*}, s_{i}\right)\right\}
$$

We have the following result, which states that there always exists a rationalizable profile of probability distributions in any production environment.

Theorem 4. There always exists a rationalizable profile of probability distributions in any environment $\left(N,\left(T_{i}\right)_{i \in N}, f\right)$. Equivalently, the game $\left(N, T,\left(u_{i}\right)_{i \in N}\right)$ associated to any production environment $\left(N,\left(T_{i}\right)_{i \in N}, f\right)$ admits a mixed strategy Nash equilibrium.

Since the game associated with a production environment is finite, the proof of this result simply follows from the proof of the existence of a mixed strategy Nash equilibrium in finite games (Nash (1951)).

We now present a natural property of the production function that ensures the existence of a pure strategy Nash equilibrium in the associated finite game.

Definition 8. Let $\left(N,\left(T_{i}\right)_{i \in N}, f\right)$ be a production environment. The function $f$ satisfies the positive interaction property (PIP) if for all $x, y \in T, f(x) \leq f(y)$ if and only if $x \preceq y$, where $x \preceq y \Leftrightarrow x_{i} \leq y_{i} \forall i \in N$.

The positive interaction property is also known as the monotonicity property in the literature. The following result states that the game associated with any environment whose production function satisfies PIP admits a pure strategy Nash equilibrium. 
Theorem 5. Let $\left(N,\left(T_{i}\right)_{i \in N}, f\right)$ be an environment such that the function $f$ satisfies PIP. Its associated game $\left(N, T,\left(u_{i}\right)_{i \in N}\right)$ admits a pure strategy Nash equilibrium.

A key implication of this result is that the non-cooperative game associated with any monotonic von Neumann-Morgernstern transferable-utility game (i.e., $T_{i}=\{0,1\}$ ) admits a pure strategy Nash equilibrium under the Shapley wage function. That equilibrium is such that each player chooses action 1. Interestingly, this finding provides a condition under which the assumption of the grand coalition can be justified or rationalized in the calculation of the Shapley value in the classical work of Shapley (1953). As shown in the examples below, relaxing this condition may lead to other equilibria emerging, which illustrates the existence of a more general set of rationalizable profiles of probability distributions in a production environment.

Example 7. Consider the production environment $\left(N,\left(T_{i}\right)_{i \in N}, f\right)$, where $N=\{1,2\}, T_{1}=\{0,1\}=T_{2}$, and the production function $f$ is defined as follows:

$$
f(x)=\left\{\begin{array}{ll}
0 & \text { if } x=(0,0) \\
-2 & \text { if } x=(1,0) \\
-4 & \text { if } x=(0,1) \\
6 & \text { if } x=(1,1)
\end{array} .\right.
$$

The Shapley payoffs derived from the associated strategic game $\left(N, T,\left(u_{1}, u_{2}\right)\right)$ are displayed in Table 6 below:

Table 6: Payoffs $\left(u_{1}(x), u_{2}(x)\right)$

\begin{tabular}{|c|c|c|c|}
\hline & \multicolumn{3}{|c|}{ Player 2} \\
\hline & strategies & $0(\beta)$ & $1(1-\beta)$ \\
\hline Player 1 & $0(\alpha)$ & $(0,0)$ & $(0,-4)$ \\
\hline & $1(1-\alpha)$ & $(-2,0)$ & $(4,2)$ \\
\hline
\end{tabular}

The profile $\pi=\left(\pi_{1}, \pi_{2}\right)$ such that $\pi_{1}(0)=\alpha, \pi_{1}(1)=1-\alpha, \pi_{2}(0)=\beta$, and $\pi_{1}(1)=1-\beta$, with $0 \leq \alpha, \beta \leq 1$, represents a profile of probability distributions over the set of pure strategies. We find that the strategic form game has two pure strategy Nash equilibria which are $(0,0)$ and $(1,1)$. The game also has a mixed strategy Nash equilibrium in which player 1 chooses the effort level 0 with probability $\frac{1}{3}$, whereas player 2 chooses the effort level 0 with probability $\frac{2}{3}$. There are therefore three rationalizable profiles of probability distributions in this game: $((1,0),(1,0)),((0,1),(0,1))$, and $\left(\left(\frac{1}{3}, \frac{2}{3}\right),\left(\frac{2}{3}, \frac{1}{3}\right)\right)$.

Example 8. Consider a production environment with three workers and two effort levels 0 and 1 . The production function is the following:

$$
f(x)=\left\{\begin{array}{lll}
1 & \text { if } & |x| \in\{2,3\} \\
0 & & \text { otherwise }
\end{array} .\right.
$$

Table 7 below displays the Shapley wage profile corresponding to each profile of effort levels: 
Table 7: Effort profile $(x)$ and Shapley wage profile $\left(\left(u_{1}(x), u_{2}(x), u_{3}(x)\right)\right)$.

\begin{tabular}{|c|c|c|c|}
\hline$x$ & $\left(u_{1}(x), u_{2}(x), u_{3}(x)\right)$ & $x$ & $\left(u_{1}(x), u_{2}(x), u_{3}(x)\right)$ \\
\hline$(0,0,0)$ & $(0,0,0)$ & $(0,1,1)$ & $\left(0, \frac{1}{2}, \frac{1}{2}\right)$ \\
\hline$(0,0,1)$ & $(0,0,0)$ & $(1,0,1)$ & $\left(\frac{1}{2}, 0, \frac{1}{2}\right)$ \\
\hline$(0,1,0)$ & $(0,0,0)$ & $(1,1,0)$ & $\left(\frac{1}{2}, \frac{1}{2}, 0\right)$ \\
\hline$(1,0,0)$ & $(0,0,0)$ & $(1,1,1)$ & $\left(\frac{1}{3}, \frac{1}{3}, \frac{1}{3}\right)$ \\
\hline
\end{tabular}

The pure strategy Nash equilibria are $(0,0,0)$ and $(1,1,1)$. We want to determine whether there exist other equilibria. Let $S_{i}$ be the set of player $i$ 's mixed strategies. Then $S_{1}=\{(\alpha, 1-\alpha): \alpha \in[0,1]\}, S_{2}=$ $\{(\beta, 1-\beta): \beta \in[0,1]\}$, and $S_{3}=\{(\gamma, 1-\gamma): \gamma \in[0,1]\}$. Consider a mixed strategy profile $s=((\alpha, 1-$ $\alpha),(\beta, 1-\beta),(\gamma, 1-\gamma))$. We derive the expected payoff at the profile $s$ as follows:

$$
\begin{aligned}
& E_{1}(s)=(1-\alpha)\left\{\frac{1}{3}(1-\gamma)\left(\frac{1}{2} \beta+1\right)+\frac{1}{2} \gamma(1-\beta)\right\}, \\
& E_{2}(s)=(1-\beta)\left\{\frac{1}{3}(1-\gamma)\left(\frac{1}{2} \alpha+1\right)+\frac{1}{2} \gamma(1-\alpha)\right\}, \\
& E_{3}(s)=(1-\gamma)\left\{\frac{1}{3}(1-\beta)\left(\frac{1}{2} \alpha+1\right)+\frac{1}{2} \beta(1-\alpha)\right\} .
\end{aligned}
$$

Player $i$ randomizes between strategies 0 and 1 if and only if he is indifferent between them; that is, $u_{i}(s \mid \alpha=$ $1)=u_{i}(s \mid \alpha=1)$. Thus, we have the following:

$$
\begin{aligned}
& E_{1}(s \mid \alpha=1)=u_{1}(s \mid \alpha=0) \text { if and only if } \gamma=\frac{\beta+2}{4 \beta-1} \\
& E_{2}(s \mid \beta=1)=u_{2}(s \mid \beta=0) \text { if and only if } \alpha=\frac{\gamma+2}{4 \gamma-1} \\
& E_{3}(s \mid \gamma=1)=u_{3}(s \mid \gamma=0) \text { if and only if } \beta=\frac{\alpha+2}{4 \alpha-1}
\end{aligned}
$$

Equations (8), (9) and (10) lead to the system: $(S) \begin{cases}\gamma=\frac{\beta+2}{4 \beta-1} & \text { (a) } \\ \alpha=\frac{\gamma+2}{4 \gamma-1} & \text { (b) } \\ \beta=\frac{\alpha+2}{4 \alpha-1} & \text { (c). }\end{cases}$

Substituting expression $\gamma$ from equation (a) into equation (b) leads to $\alpha=\beta(d)$. Rewriting equation (c) under condition $(d)$ gives equation $2 \alpha^{2}-\alpha-1=0(e)$. The latter equation has two roots: $\alpha=-\frac{1}{2}$ and $\alpha=1$. Since $\alpha$ is positive, we can conclude that there are only two rationalizable probability distributions in this game that correspond to the pure strategy Nash equilibria $(0,0,0)$ and $(1,1,1)$.

\section{An Application to Contagion in a Networked Economy}

In this section, we provide an application of the a priori and the Bayesian Shapley values to contagion in a networked economy. We show how these values can be used to determine the contribution of each agent (or node) to contagion. In particular, this application is illustrated using fidelity networks (Pongou (2010), Pongou and Serrano (2013)). 
Consider an economy $\mathcal{M}$ involving agents who freely form and sever bilateral links according to their preferences. Assume that rational behavior is captured by a certain equilibrium notion (e.g., pairwise stability, stochastic stability, etc.). Such an economy may have multiple equilibria. Denote by $\mathcal{E}(\mathcal{M})$ the set of its equilibria. Our goal is twofold. First, we would like to evaluate the expected contribution of each agent (or node) to the spread of a random infection that might hit the economy. Second, given an observed contagion level, we would like to determine the contribution of each agent to that level.

To illustrate these notions, consider a fidelity economy $\mathcal{M}$ involving two men $m_{1}$ and $m_{2}$ and two women $w_{1}$ and $w_{2}$. Man $m_{1}$ desires one female partner; man $m_{2}$ desires two female partners; and each woman desires only one male partner. ${ }^{5}$ A network is a collection of links between men and women. This economy can be represented by an uncertain production environment $\left(N,\left(T_{i}\right)_{i \in N}, \widetilde{f}, \pi\right)$, where $N=\left\{m_{1}, m_{2}, w_{1}, w_{2}\right\}$; $T_{m_{1}}=\left\{\emptyset,\left\{w_{1}\right\},\left\{w_{2}\right\}\right\} ; T_{m_{2}}=\left\{\emptyset,\left\{w_{1}\right\},\left\{w_{2}\right\},\left\{w_{1}, w_{2}\right\}\right\} ; T_{w_{1}}=\left\{\emptyset,\left\{m_{1}\right\},\left\{m_{2}\right\}\right\} ; T_{w_{2}}=\left\{\emptyset,\left\{m_{1}\right\},\left\{m_{2}\right\}\right\}, \tilde{f}$ is a contagion function (see below), and $\pi$ is a probability distribution over the set of input profiles.

For simplicity, denote $\left\{w_{1}\right\}$ by $1,\left\{w_{2}\right\}$ by $2,\left\{m_{1}\right\}$ by $3,\left\{m_{2}\right\}$ by $4,\left\{w_{1}, w_{2}\right\}$ by 5 , and the empty set $\emptyset$ by 0 . The input profile $x=(1,5,4,4)$, for instance, means that $m_{1}$ desires to match with $w_{1}, m_{2}$ desires to match with $w_{1}$ and $w_{2}, w_{1}$ desires to match with $m_{2}$, and $w_{2}$ desires to match with $m_{2}$. A match between two individuals occurs if it is desired by both. The input profile $x=(1,5,4,4)$ therefore leads to the network $g_{2}$ in Figure 1 below. If at an input profile $x, x_{i}=0$ for an individual $i$, it means that $i$ wants no partner. It follows from this interpretation that each input profile $x \in T=\prod_{i \in N} T_{i}$ is a network.

Assume that rationality is captured by the notion of pairwise stability as defined in Pongou and Serrano (2013, 2016). A network $g$ is pairwise stable if: (i) no individual has an incentive to sever an existing link in which he is involved; and (ii) no pair of a man and a woman has an incentive to form a new link while at the same time severing some of the existing links in which they are involved. There are 8 possible networks in this economy, but only 3 of them are pairwise stable. These equilibrium networks are represented by the graphs below:

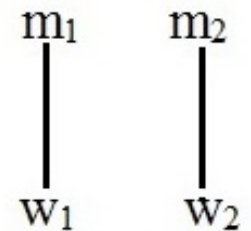

$g_{1}$

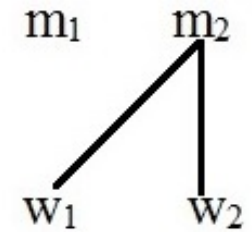

$g_{2}$

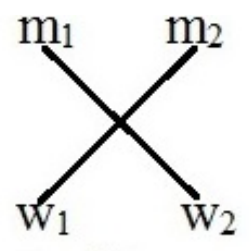

$g_{3}$

Figure 1: pairwise stable networks

The contagion function is the contagion potential of a network (Pongou (2010)). To define this function, let $g$ be an $n$-person network that has $k$ components, where a component is a maximal set of agents who are

\footnotetext{
${ }^{5}$ Gale and Shapley (1962) pioneered the analysis of two-sided economies. But our models differ in their scopes and applications.
} 
directly or indirectly connected in $g$; and $n_{j}$ the number of individuals in the $j^{\text {th }}$ component $(1 \leq j \leq k)$. Pongou (2010) (see also Pongou and Serrano $(2013,2016)$ ) shows that if a random individual is infected with a virus (e.g., HIV), and if that individual infects his/her partners who also infect their other partners and so on, the fraction of infected people is given by the contagion potential of $g$, which is:

$$
\mathcal{P}(g)=\frac{1}{n^{2}} \sum_{j=1}^{k} n_{j}^{2} .
$$

We can also derive the part of contagion for which agents are collectively responsible in $g$. It is:

$$
\widetilde{f}(g)=\mathcal{P}(g)-\frac{1}{n}
$$

This expression is justified because each individual in $g$ is exogenously infected with probability $\frac{1}{n}$, and agents are not responsible for exogenous infections.

Following this formula, we calculate the contagion potential of each equilibrium network:

$$
\mathcal{P}\left(g_{1}\right)=\frac{1}{4^{2}}\left(2^{2}+2^{2}\right)=0.5, \mathcal{P}\left(g_{2}\right)=0.625, \text { and } \mathcal{P}\left(g_{3}\right)=0.5 \text {. }
$$

Our goal is being able to determine the responsibility of each agent for the level $\widetilde{f}(g)$ of transmitted contagion in a network $g$. Let $S$ be a set of agents. We denote by $g(S)$ the restriction of the network $g$ to $S$. This restriction is obtained by severing all the links involving agents in $N \backslash S$. Also, let $i$ be an individual. We denote by $g(S)+i$ the network $g(S \cup\{i\})$ obtained from $g(S)$ by connecting $i$ to all the agents in $S$ to whom $i$ is connected in the network $g$. The a priori Shapley value of each individual $i$ in the network $g$ is:

$$
\mathcal{P}_{i}(g)=\sum_{S \subseteq N, i \notin S} \frac{s !(n-s-1) !}{n !}[\mathcal{P}(g(S)+i)-\mathcal{P}(g(S))], s=|S| .
$$

The $a$ priori Shapley value for each agent in each equilibrium network is given by Table 8 .

Table 8: Individual contagion potential in equilibrium networks

\begin{tabular}{|c|c|c|c|c|}
\hline Stable networks $(g)$ & $\mathcal{P}_{m_{1}}(g)$ & $\mathcal{P}_{m_{2}}(g)$ & $\mathcal{P}_{w_{1}}(g)$ & $\mathcal{P}_{w_{2}}(g)$ \\
\hline$g_{1}$ & $\frac{1}{16}$ & $\frac{1}{16}$ & $\frac{1}{16}$ & $\frac{1}{16}$ \\
\hline$g_{2}$ & 0 & $\frac{8}{48}$ & $\frac{5}{48}$ & $\frac{5}{48}$ \\
\hline$g_{3}$ & $\frac{1}{16}$ & $\frac{1}{16}$ & $\frac{1}{16}$ & $\frac{1}{16}$ \\
\hline
\end{tabular}

Remark that in each network $g$, the contagion potentials (Shapley values) of agents sum up to $\widetilde{f}(g)$, so that the contagion potential of each agent measures his/her responsibility for the transmitted contagion.

Only equilibrium networks are likely to form. Therefore, the a priori Shapley value of an agent $i$ to contagion in the economy $\mathcal{M}$ is:

$$
\mathcal{P}_{i}(\mathcal{M})=\sum_{g \in \mathcal{E}(\mathcal{M})} \pi(g) \mathcal{P}_{i}(g) .
$$

Remark that we do not yet know what the probability distribution $\pi$ is. We can assume that each equilibrium network is equally likely to form. But we can also assume a network formation process following random 
pairwise meetings as follows. Assume that men and women meet randomly and decide whether to form a link or not. For instance, consider a possible order of meeting $r=\left(\left\{m_{1}, w_{1}\right\},\left\{m_{1}, w_{2}\right\},\left\{m_{2}, w_{1}\right\},\left\{m_{2}, w_{2}\right\}\right)$, interpreted as follows: agents $m_{1}$ and $w_{1}$ meet first; then agents $m_{1}$ and $w_{2}$ meet; then agents $m_{2}$ and $w_{1}$ meet; finally agents $m_{2}$ and $w_{2}$ meet. At each meeting, the meeting agents form a link if they mutually desire each other and have not reached yet their optimal number of partners. The order meeting $r$ above will lead to the network $g_{1}$. There are 24 meeting orders in total. If we assume each order to be equally likely, then we find the following probability of formation for each network: $\pi\left(g_{1}\right)=0.375, \pi\left(g_{2}\right)=0.25$, and $\pi\left(g_{3}\right)=0.375$. Using this probability distribution, we compute the contagion potential of each individual in the mating economy $\mathcal{M}$ as follows:

$$
A S V_{m_{1}}(\mathcal{M})=0.046875, A S V_{m_{2}}(\mathcal{M})=0.0885, \text { and } A S V_{w_{1}}(\mathcal{M})=A S V_{w_{2}}(\mathcal{M})=0.0729 .
$$

Clearly, we see that $m_{2}$ has the highest contagion potential, which is justified by the fact that his demand for partners is the highest.

Now, assume that the prevalence of the infection in the population is $25 \%$. We are interested in determining the responsibility of each agent for the contagion. There exist only two networks that lead to a contagion prevalence of $25 \%$, namely $g_{1}$ and $g_{3}$ (recall that $\left.\tilde{f}\left(g_{1}\right)=\tilde{f}\left(g_{3}\right)=\mathcal{P}\left(g_{1}\right)-0.25=\mathcal{P}\left(g_{3}\right)-0.25=0.25\right)$. The Bayesian Shapley value is therefore:

$$
B S A_{i}=\frac{\pi\left(g_{1}\right) \mathcal{P}_{i}\left(g_{1}\right)+\pi\left(g_{3}\right) \mathcal{P}_{i}\left(g_{3}\right)}{\pi\left(g_{1}\right)+\pi\left(g_{3}\right)}, \text { for } i \in\left\{m_{1}, m_{2}, w_{1}, w_{2}\right\}
$$

Using the information above, we have : $B S A_{m_{1}}=B S A_{m_{2}}=B S A_{w_{1}}=B S A_{w_{2}}=0.0625$.

Importantly, whereas the a priori Shapley value depends on the structure of economy, the Bayesian Shapley value depends on the structure of the equilibrium network that possibly realizes. As shown by the example above, these values can be very different.

\section{Conclusion}

In this paper we address the problem of valuing inputs in a production environment in which input supply is uncertain. We solve this problem in a new class of economic environments and show several applications. Inputs can be workers in a firm, risk factors for a disease, securities in a financial market, or agents (or nodes) in a networked economy. Using three intuitive axioms, we uniquely characterize a valuation scheme that solves the valuation problem. We call this scheme the a priori Shapley value. Second, we address the problem of valuing inputs a posteriori, that is, after the realization of the output. Using a Bayesian updating procedure to determine the posterior probability distributions of inputs enables us to define the Bayesian Shapley value. We also provide a set of axioms that uniquely characterize this value. Third, we ask whether uncertainty can be rationalized in a world in which inputs are rational workers supplying labor in a noncooperative production game in which payoffs are determined by the Shapley wage function. We find that 
probability distributions over labor supply that can be supported as mixed strategy Nash equilibria always exist. We also provide an intuitive condition under which a pure strategy Nash equilibrium exists. These findings rationalize both random and non-random labor supply in organizations. We show several applications of the a priori and the Bayesian Shapley values, including an application to networked economies. 


\section{References}

Aguiar, V. H., Pongou, R., and Tondji, J. B. (2016). Measuring and decomposing the distance to the Shapley wage function with limited data. Mimeo.

Allen, F., and Gale, D. (2000). Financial contagion. Journal of political economy, 108(1), 1-33.

Battiston, S., Gatti, D. D., Gallegati, M., Greenwald, B., and Stiglitz, J. E. (2012). Liaisons dangereuses: Increasing connectivity, risk sharing, and systemic risk. Journal of Economic Dynamics and Control, $36(8), 1121-1141$.

Freixas, J. (2005). The Shapley-Shubik power index for games with several levels of approval in the input and output. Decision Support Systems, 39, 185-195.

Gale, D., and Shapley, L., (1962). College admissions and the stability of marriage. American Mathematical Monthly 69, 9-15.

Hsiao, Chih-Ru, and Raghavan T.E.S. (1993). Shapley value for multichoice cooperative games, I. Games and Economics Behavior, 5, 240-256.

Lin, J. (2016). Using Weighted Shapley Values to Measure the Systemic Risk of Interconnected Banks. Pacific Economic Review.

Nash, F. J. (1951). Non-cooperative games. The Annals of Mathematics, 54(2), 286-295.

Pongou, R. (2010). The economics of fidelity in network formation. PhD Dissertation, Brown University.

Pongou, R., and Serrano, R. (2013). Fidelity networks and long-run trends in HIV/AIDS gender gaps. The American Economic Review, 103(3), 298-302.

Pongou, R., and Serrano, R. (2016). Volume of trade and dynamic network formation in two-sided economies. Journal of Mathematical Economics, 63, 147-163.

Pongou, R., and Tondji, J-B. (2016). Non-cooperative games with Shapley payoffs. Working paper.

Roth, A. (1988). The Shapley value: essays in honor of Lloyd S. Shapley, Cambridge University Press.

Serrano, R. (2013). Lloyd Shapley's matching and game theory. Scandinavian Journal of Economics, 115, 599-618.

Shapley, L. (1953). A Value for $n$-person games. In Contributions to the Theory of Games, 2, 307-317.

Shapley, L., and Shubik, M. (1967). Ownership and the production function. The Quarterly Journal of Economics, 88-111.

Young, H. P. (1985). Monotonic solutions of cooperative games. International Journal of Game Theory, 14 $(2), 65-72$ 


\section{Appendix}

In order to prove Theorem 1, we need to establish some preliminary results. Define the following production function $f_{x}$ for each $x \in T$ by:

$$
f_{x}(y)=\left\{\begin{array}{lll}
1 & \text { if } & x \unlhd y \\
0 & \text { if } & x \notin y
\end{array}\right.
$$

The following lemma, proved in Aguiar et al.(2016), shows that every production function is a linear combination of these basic functions.

Lemma 1. Any production function is a linear combination of the production functions $f_{x}$ :

$$
f=\sum_{x \in T ; x \neq 0} c_{x}(f) f_{x}, \text { where } c_{x}(f)=\sum_{x^{\prime} \unlhd x}(-1)^{|x|-\left|x^{\prime}\right|} f\left(x^{\prime}\right)
$$

In order to prove Theorem 1, we also need to show that the a priori Shapley value satisfies the probabilistic additivity property defined below.

Definition 9. A valuation procedure $\phi$ is probabilistically additive if for any uncertain production environment $\mathcal{P}=\left(N,\left(T_{i}\right)_{i \in N}, f, \pi\right)$ and $\mathcal{P}^{\prime}=\left(N,\left(T_{i}\right)_{i \in N}, g, \pi\right), \phi(f+g)=\phi(f)+\phi(g)$.

We now state the following result.

Lemma 2. The a priori Shapley value $A S V$ is probabilistically additive.

Proof. Let $\mathcal{P}=\left(N,\left(T_{i}\right)_{i \in N}, f, \pi\right)$ and $\mathcal{P}^{\prime}=\left(N,\left(T_{i}\right)_{i \in N}, g, \pi\right)$ be two production environments. Consider an input $i \in N$; then we have:

$$
A S V_{i}(f+g)=\sum_{x \in T} \pi(x)\left\{A S V_{i}^{f+g}(x)\right\}
$$

where:

$$
\begin{aligned}
A S V_{i}^{f+g}(x) & =\sum_{a \triangleleft_{0}^{i} x} \frac{(|a|) !(|x|-|a|-1) !}{(|x|) !}\left\{(f+g)\left[a+x_{i} e_{i}\right]-(f+g)[a]\right\} \\
& =\sum_{a \triangleleft_{0}^{i} x} \frac{(|a|) !(|x|-|a|-1) !}{(|x|) !}\{m c(, i, f, a, x)\} \\
& +\sum_{a \triangleleft_{0}^{i} x} \frac{(|a|) !(|x|-|a|-1) !}{(|x|) !}\{m c(i, g, a, x)\} \\
& =A S V_{i}^{f}(x)+A S V_{i}^{g}(x) .
\end{aligned}
$$

We can conclude that $A S V_{i}(f+g)=\sum_{x \in T} \pi(x) A S V_{i}^{f}(x)+\sum_{x \in T} \pi(x) A S V_{i}^{g}(x)=A S V_{i}(f)+A S V_{i}(g)$, and $A S V$ satisfies the probabilistic additivity property. 
Now, we are ready to prove Theorem 1.

\section{Proof of Theorem 1}

\section{A. Sufficiency}

In this part of the proof, we show that the a priori Shapley value $A S V$ satisfies the axioms of probabilistic symmetry, marginality, and probabilistic efficiency.

Let $\mathcal{P}=\left(N,\left(T_{i}\right)_{i \in N}, f, \pi\right)$ be an uncertain production environment.

1. Probabilistic symmetry: Let $i$ and $j$ be two probabilistically symmetric inputs. Then:

$$
A S V_{i}(f)=\sum_{x \in T, x_{i}=x_{j}} \pi(x) A S V_{i}(x)+\sum_{x \in T, x_{i}>x_{j}} \pi(x) A S V_{i}(x)+\sum_{x \in T, x_{i}<x_{j}} \pi(x) A S V_{i}(x) .
$$

The a priori Shapley value of input $i$ at a given input profile $x$ is given by:

$$
\begin{aligned}
A S V_{i}(x) & =\sum_{a \triangleleft_{0}^{i} x} \varphi(a, x)\left[f\left(a+x_{i} e_{i}\right)-f(a)\right] \\
& =\sum_{a \triangleleft_{0}^{i} x \text { and } a_{j}=0} \varphi(a, x)\left[f\left(a+x_{i} e_{i}\right)-f(a)\right]+\sum_{a \triangleleft_{0}^{i} x \text { and } a_{j} \neq 0} \varphi(a, x)\left[f\left(a+x_{i} e_{i}\right)-f(a)\right] \\
& =\sum_{b \triangleleft_{0}^{j} x \text { and } b_{i}=0} \varphi(b, x)\left[f\left(a+x_{i} e_{j}\right)-f(a)\right] \\
& +\sum_{b \triangleleft_{0}^{j} x \text { and } b_{i} \neq 0, b=a-x_{j} e_{j}+x_{j} e_{i}} \varphi(b, x)\left[f\left(b+x_{i} e_{j}\right)-f(b)\right] .
\end{aligned}
$$

Note that for any $x, y \in T$ such that: $\left(x_{i}=a\right.$ and $\left.x_{j}=b\right),\left(y_{j}=a\right.$ and $\left.y_{i}=b\right)$, and $y_{k}=x_{k}$ for any $k \neq i, j$, we have $A S V_{i}(x)=A S V_{j}(y)$. Moreover, given that $\pi_{i}=\pi_{j}$, we have $\pi(x)=\pi(y)$. Since $A S V_{i}(x)=A S V_{j}(x)$ for all $x$ such that $x_{i}=x_{j}$, we can conclude that:

$$
\begin{aligned}
A S V_{i}(\mathcal{P}) & =\sum_{x \in T, x_{i}=x_{j}} \pi(x) A S V_{j}(x)+\sum_{x \in T, x_{j}>x_{i}} \pi(x) A S V_{j}(x)+\sum_{x \in T, x_{j}<x_{i}} \pi(x) A S V_{j}(x) \\
& =A S V_{j}(\mathcal{P}) .
\end{aligned}
$$

2. Marginality: consider a production environment $\mathcal{P}^{\prime}=\left(N,\left(T_{i}\right)_{i \in N}, g, \pi\right)$ such that $m c(i, f, a, x) \geq$ $m c(i, g, a, x)$ for all $i \in N, x, a \in T$ and $a \triangleleft_{0}^{i} x$. By the definition of the a priori Shapley value, we have $A S V_{i}^{f}(x) \geq A S V_{i}^{g}(x)$. Hence, $\sum_{x \in T} \pi(x) A S V_{i}^{f}(x) \geq \sum_{x \in T} \pi(x) A S V_{i}^{g}(x)$, that is $A S V_{i}(f) \geq A S V_{i}(g)$, or equivalently, $A S V_{i}(\mathcal{P}) \geq A S V_{i}\left(\mathcal{P}^{\prime}\right)$.

3. Probabilistic efficiency: for the production environment $\mathcal{P}$, we have:

$$
\begin{aligned}
\sum_{i \in N} A S V_{i}(\mathcal{P}) & =\sum_{i \in N} \sum_{x \in T} \pi(x) A S V_{i}(x) \\
& =\sum_{x \in T} \pi(x)\left\{\sum_{i \in N} A S V_{i}(x)\right\} \\
& =\sum_{x \in T} \pi(x) f(x), \text { since } \sum_{i \in N} A S V_{i}(x)=f(x) .
\end{aligned}
$$

Hence, $A S V$ satisfies the probabilistic efficiency axiom. This concludes the first part of the proof. 


\section{B. Necessity}

In this part of the proof, we prove the uniqueness of the a priori Shapley value. Consider another allocation procedure $\phi$ which satisfies the axioms of probabilistic efficiency, marginality and probabilistic symmetry. We would like to show that, for any production environment $\mathcal{P}=\left(N,\left(T_{i}\right)_{i \in N}, f, \pi\right), \phi_{i}(f)=A S V_{i}(f)$ for each input $i \in N$.

1. Consider a production function $f$ which is identically zero on all input profiles. Then

$$
m c(i, f, a, x)=0 \text { for all } x, a \in T \text { such that } a \triangleleft x \text {; . }
$$

Given that $\phi$ satisfies the probabilistic symmetry axiom, we have $\phi_{i}(f)=\phi_{j}(f)$ for $j \neq i$, and by the probabilistic efficiency axiom, we have:

$$
\sum_{i \in N} \phi_{i}(f)=\sum_{x \in T} \pi(x) f(x)=0
$$

Hence, $\phi_{i}(f)=0$ for each input $i \in N$. We can show in the same manner that for any production function $f$ on $T$, an input profile $x \in T$, and any $i \in N$ :

$$
\text { if } m c(i, f, a, x)=0 \text { for all } x, a \in T \text { such that } a \triangleleft x \text {, then } \phi_{i}(f)=0 \text {. }
$$

If $f \equiv 0$, we also have $A S V_{i}(f)=\sum_{x \in T} \pi(x) A S V_{i}(x)=0$, since $A S V_{i}(x)=0$ for all $x$.

2. Let $f$ be a production function, then by using Lemma 1, we can write:

$$
f(y)=\sum_{x \in T, x \neq 0} c_{x}(f) f_{x}(y)
$$

By the definition of $f_{x}$, the latter expression (equation (16)) can be rewritten as:

$$
f(y)=\sum_{x \in T, x \neq 0, x \unlhd y} c_{x}(f) f_{x}(y)=\sum_{x \in T,} c_{x \neq 0, x \unlhd y}(f) .
$$

It follows that for $i \in N$ :

$$
\phi_{i}(f)=\phi_{i}\left\{\sum_{x \in T, x \neq 0, x \unlhd y} c_{x}(f) f_{x}(y)\right\}=\phi_{i}\left\{\sum_{x \in T, x \neq 0, x \unlhd y} c_{x}(f)\right\} .
$$

Define the index $I$ of the production function $f$ to be the minimum number of non-zero terms in some expression for $f$ of the form established in Lemma 1. The theorem is proved by induction on $I$.

a) If $I=0$, then $f \equiv 0$. By the probabilistic efficiency requirement, we have:

$$
\sum_{i \in N} \phi_{i}(f)=\sum_{x \in T} \pi(x) f(x)=0 .
$$

Using the probabilistic symmetry axiom, we have $|N| \phi_{i}(f)=0$, which implies that $\phi_{i}(f)=0$. Then, $\phi_{i}(f)$ should coincide with $A S V_{i}(f)$, since $A S V_{i}(x)=0$ for all $x$. 
b) If $I=1$, then $f=c_{x}(f) f_{x}$ for some $x \in T$. Thus, $\phi_{i}(f)=\phi_{i}\left(c_{x}(f) f_{x}\right)$. Consider $X_{x}=$ $\left\{l \in N: x_{l}>0\right\}$ and let $i \notin X_{x}$. For $a, x \in T$ such that $a \triangleleft_{0}^{i} x$, we have $f\left(a+x_{i} e_{i}\right)-f(a)=0$, i.e $m c(i, f, a, x)=0$. It follows from equation (15) and from the fact that $\phi_{i}$ satisfies the marginality axiom that $\phi_{i}(f)=0$. Moreover, $A S V_{i}(x)=0$ for all $x$, leading to $A S V_{i}(f)=0$. Let $i, j \in N$ such that $i, j \in X_{x}$. Let us show that $i$ and $j$ are probabilistically symmetric.

- Consider $y \in T$ such that $y_{i}=y_{j}=0$. We have $f(y)=c_{x}(f)$ if $x \unlhd y$. Since $i, j \in X_{x}$, it means that $x_{i}>0$ and $x_{j}>0$. Then, $x \not y$ and $f(y)=f\left(\tau_{i j}(y)\right)=0$.

- Consider $y \in T$ such that $\left(y_{i}=0\right.$ and $\left.y_{j} \neq 0\right)$ or $\left(y_{j}=0\right.$ and $\left.y_{i} \neq y_{j}\right)$. For the same reason stated above, $f(y)=f\left(\tau_{i j}(y)\right)=0$.

We can conclude that inputs $i$ and $j$ are probabilistically symmetric. Using the axioms of probabilistic symmetry and probabilistic efficiency, we have:

$$
|x| \phi_{i}(f)=\sum_{x \unlhd y, y \in T} \pi(y) c_{x}(f)
$$

so that,

$$
\phi_{i}(f)=\frac{\sum_{x \unlhd y, y \in T} \pi(y) c_{x}(f)}{|x|}=A S V_{i}(f) .
$$

c) Assume now that $\phi$ is the value $A S V$ whenever the index of $f$ is at most $I$ and let $f$ have index $I+1$, with:

$$
f=\sum_{k=1}^{I+1} c_{x^{k}}(f) f_{x^{k}}, \text { all } c_{x^{k}} \neq 0, \text { and } x^{k} \in T .
$$

For $k \in\{1,2, \ldots, I+1\}$, consider:

$$
X_{k}=\left\{l \in N: x_{l}^{k}>0\right\}, X=\bigcap_{k=1}^{I+1} X_{k}, \text { and assume } i \notin X .
$$

Define the following production function:

$$
g=\sum_{k: i \in X_{k}} c_{x^{k}}(f) f_{x^{k}} .
$$

The index of $g$ is at most $I$. Let $x, a \in T$ such that $a \triangleleft_{0}^{i} x$. Then $f\left(a+x_{i} e_{i}\right)-f(a)=g\left(a+x_{i} e_{i}\right)-g(a)$. Hence, using the marginality axiom, $\phi_{i}(f)=\phi_{i}(g)$. By induction, we have:

$$
\phi_{i}(f)=\sum_{k: i \in X_{k}}\left\{\frac{\sum_{y \in T, x^{k} \unlhd y} \pi(y) c_{x^{k}}(f)}{\left|x^{k}\right|}\right\},
$$

which is the value of $A S V_{i}(f)$ by using the aforementioned properties.

It remains to show that $\phi_{i}(f)=A S V_{i}(f)$ when $i \in X$. By the probabilistic symmetry axiom, $\phi_{i}(f)$ is a constant $\epsilon$ for all members of $X$; likewise the value $A S V_{i}(f)$ is some constant $\epsilon^{\prime}$ for all members of $X$. Since both allocations sum to $\sum_{x \in T} \pi(x) f(x)$ and are equal for all $i \in X$, it follows that $\epsilon=\epsilon^{\prime}$. 
Proof of Theorem 2: The proof follows the same reasoning as that of Theorem 1.

Proof of Theorem 3: Consider a production environment $\mathcal{P}=\left(N,\left(T_{i}\right)_{i \in N}, f, \pi\right)$. In order to establish the proof, it suffices to show that the value $U$ satisfies the axioms of marginality, probabilistic symmetry, and probabilistic efficiency. We recall that:

$$
U_{i}(f)=\sum_{Y \in \mathcal{R}_{f}} P(Y) B S A_{i}(Y),
$$

where $\mathcal{R}_{f}=\{Y \in \mathbb{R}: \exists x \in T, f(x)=Y\}$ is the set of realizable outputs and $Y_{f}=\{x \in T: f(x)=Y\}$ for $Y \in \mathcal{R}_{f}$.

1. First, let us show that $U$ satisfies the probabilistic efficiency axiom. Summing up $U_{i}(f)$ yields:

$$
\sum_{i \in N} U_{i}(f)=\sum_{Y \in \mathcal{R}_{f}} P(Y)\left\{\sum_{i \in N} B S A_{i}(Y)\right\}
$$

Now, for $Y \in \mathcal{R}_{f}$, we have:

$$
\begin{aligned}
\sum_{i \in N} B S A_{i}(Y) & =\sum_{i \in N}\left\{\frac{\sum_{x \in Y_{f}} \pi(x) A S V_{i}(x)}{\sum_{x \in Y^{f}} \pi(x)}\right\} \\
& =\frac{\sum_{x \in Y_{f}} \pi(x)\left\{\sum_{i \in N} A S V_{i}(x)\right\}}{\sum_{x \in Y_{f}} \pi(x)} \\
& =\frac{\sum_{x \in Y_{f}} \pi(x) f(x)}{\sum_{x \in Y_{f}} \pi(x)} \text { since } \sum_{i \in N} A S V_{i}(x)=f(x) \\
& =\frac{\sum_{x \in Y_{f}} \pi(x) Y}{\sum_{x \in Y_{f}} \pi(x)} \text { since for each } x \in Y_{f}, f(x)=Y \\
& =Y .
\end{aligned}
$$

Hence, it follows that:

$$
\sum_{i \in N} U_{i}(f)=\sum_{Y \in \mathcal{R}_{f}}\{P(Y) Y\}=\sum_{Y \in \mathcal{R}_{f}}\left\{\sum_{x \in Y_{f}} \pi(x) f(x)\right\},
$$

since $P(Y)=\sum_{x \in Y_{f}} \pi(x)$ and $f(x)=Y$ for each $x \in Y_{f}$. For any $x \in T$, there exists $Y \in \mathcal{R}_{f}$ such that $f(x)=Y$. Also, for any $Y \in \mathcal{R}_{f}$, there exists $x \in T$ such that $f(x)=Y$. Therefore,

$$
\sum_{Y \in \mathcal{R}_{f}}\left\{\sum_{x \in Y_{f}} \pi(x) f(x)\right\}=\sum_{x \in T} \pi(x) f(x) .
$$

We conclude that $U$ satisfies the probabilistic efficiency axiom.

2. Second, we show that $U$ satisfies the marginality axiom. For any production environment $\mathcal{P}=\left(N,\left(T_{i}\right)_{i \in N}, f, \pi\right)$ and any input $i \in N$, the function $U_{i}$ can be rewritten as:

$$
U_{i}(f)=\sum_{Y \in \mathcal{R}_{f}}\left\{\sum_{x \in Y_{f}} \pi(x) A S V_{i}^{f}(x)\right\} .
$$


Let $\mathcal{P}^{\prime}=\left(N,\left(T_{i}\right)_{i \in N}, g, \pi\right)$ be another production environment such that $m c(i, f, a, x) \geq m c(i, g, a, x)$ for all $i \in N, a, x \in T$ with $a \triangleleft_{0}^{i} x$. By the definition of the a priori Shapley value $A S V$, we have $A S V^{f} i(x) \geq A S V_{i}^{g}(x)$. Hence, by summation, we have $U_{i}(f) \geq U_{i}(g)$.

3. Third, we show that $U$ satisfies the probabilistic symmetry axiom. Let $i$ and $j$ be two probabilistically symmetric inputs. Then by Theorem 1, we have $A S V_{i}(x)=A S V_{j}(x)$ for any given input profile $x \in T$. Hence, by the definition of the value $U$, we conclude that $U_{i}(f)=U_{j}(f)$.

Since the a priori Shapley value is the unique scheme which simultaneously satisfies the axioms of probabilistic symmetry, marginality, and probabilistic efficiency, we conclude from Theorem 1 that the two values coincide.

Proof of Theorem 4: Since the game $\left(N, T,\left(u_{i}\right)_{i \in N}\right)$ associated to a production environment $\left(N,\left(T_{i}\right)_{i \in N}, f\right)$ is finite, the proof of the existence of a mixed strategy Nash equilibrium in that game follows from Nash (1951).

Proof of Theorem 5: We have $N=\{1,2, \ldots, n\}, T_{i}=\left\{0,1,2, \ldots, t_{i}\right\}$ for each player $i$, and $u=\left(u_{i}\right)_{i \in N}$, with $u_{i}(x)=A S V_{i}(x)$. Show that $\bar{x}=\left(t_{1}, t_{2}, \ldots, t_{n}\right)$ is a Nash equilibrium. Without loss of generality, we show that player 1 has no incentive to deviate from $\bar{x}$. Assume that he unilaterally deviates to an input level $t<t_{1}$. The resulting strategy profile will be $\bar{x}^{\prime}=\left(t, t_{2}, t_{3}, \ldots, t_{n}\right)$, and player 1 will gain:

$$
u_{1}\left(\bar{x}^{\prime}\right)=\sum_{x \triangleleft 1_{0}^{1} \bar{x}^{\prime}} \varphi\left(x, \bar{x}^{\prime}\right)\left[f\left(x+t e_{1}\right)-f(x)\right] .
$$

Let us show that $u_{1}(\bar{x}) \geq u_{1}\left(\bar{x}^{\prime}\right)$. We have: $u_{1}(\bar{x})=\sum_{x \triangleleft 1} \varphi(x, \bar{x})\left[f\left(x+t_{1} e_{1}\right)-f(x)\right]$. Notice that $x+t e_{1} \preceq$ $x+t_{1} e_{1}$ for all $x$ such that $x_{1}=0$. It follows from the fact that the production function $f$ satisfies PIP that $f\left(x+t_{1} e_{1}\right) \geq f\left(x+t e_{1}\right)$ for all $x$ such that $x_{1}=0$. It immediately follows that $u_{1}(\bar{x}) \geq u_{1}\left(\bar{x}^{\prime}\right)$. We conclude that player 1 has no incentive to deviate unilaterally from $\bar{x}$. We can show in a similar manner that no other player has an incentive to unilaterally deviate from $\bar{x}$, leading to the conclusion that $\bar{x}$ is a pure strategy Nash equilibrium. 